\title{
The Regulatory Role of PRRXI in Cancer Epithelial-Mesenchymal Transition
}

\author{
Wenjiao Du (iD ${ }^{1, *}$ \\ Xinchang Liu ${ }^{2, *}$ \\ Man Yang ${ }^{2}$ \\ Weipeng Wang ${ }^{2}$ \\ Jing Sun (D)
}

'Institute of Medical Biotechnology, Suzhou Vocational Health College, Suzhou, Jiangsu, 215009, People's Republic of China; ${ }^{2}$ Center for Drug Metabolism and Pharmacokinetics, College of Pharmaceutical Sciences, Soochow University, Suzhou, 215123, People's Republic of China

*These authors contributed equally to this work
Correspondence: Jing Sun

Institute of Medical Biotechnology,

Suzhou Vocational Health College,

Suzhou, Jiangsu, 2 I5009, People's Republic

of China

Email jsun@szhct.edu.cn

\section{Weipeng Wang}

Center for Drug Metabolism and

Pharmacokinetics, College of

Pharmaceutical Sciences, Soochow

University, Wenjing Road, Suzhou

Industrial Park, Suzhou, 215I23, People's

Republic of China

Email wangweipeng@suda.edu.cn

\begin{abstract}
PRRX1 (paired related homeobox 1), a member of the paired homeobox family, exhibits an important role in tumor. It is closely correlated to the occurrence of epithelialmesenchymal transition (EMT). PRRX1 is an important transcription factor regulating EMT and plays an important role in tumor progression. In the process of tumor metastasis, PRRX1 mainly regulates the occurrence of EMT in tumor cells through TGF- $\beta$ signaling pathway, Wnt/ $\beta$-catenin signaling pathway and Notch signaling pathway. PRRX1 is not only closely related to the tumor cell stemness but also involved in miRNA regulation of EMT. Therefore, PRRX1 may be a target for inhibiting the proliferation, metastasis and stemness of tumor cells. The current review provides a systemic profile of the regulatory role of PRRX1 in cancer epithelial-mesenchymal transition.
\end{abstract}

Keywords: PRRX1, EMT, tumor

\section{Introduction}

Homeobox genes are highly conserved and are primary regulators in the development of animals, fungi, and plants. The Homeobox gene contains about 180 nucleotides, which encodes a 60-amino-acid DNA-binding motif named the homeobox domain. The homeobox genes mostly encode transcription factors and function as activators or repressors of the other genes. ${ }^{1}$ In 1975, Garcia-Bellido proposed that homeobox genes could directly activate genes that control morphogenesis. ${ }^{2}$ He called these downstream targets the "realizator" genes, defining them as genes that determine processes such as cell shape or phenotype, adhesion, migration, and rate of mitosis or apoptosis. Studies have confirmed that homeobox genes control the "realizator" genes mainly through regulating other transcription factors and signaling molecules. ${ }^{1}$ It is also found that the downstream target genes are activated or inhibited by the homeobox genes, and play an important role in the regulation of proliferation, differentiation, and apoptosis of cells. ${ }^{3}$

PRRX1 is a transcription factor belonging to the paired homeobox family, and the gene is located in the nucleus in chromosome 1q24. And there are two main isoforms of PRRX1 called PRRX1 isoform a and b. PRRX1a, usually called PRRX1, is the canonical isoform of PRRX1. ${ }^{4}$ PRRX1a is a transcription activator and contains an OAR (otp, aristaless, and rax) domain and a homeobox DNAbinding domain. The OAR domain is a 15-amino-acid region shared by orthopedia homeobox (OTP), aristaless related homeobox (ARX), and retina and anterior neural fold homeobox (RAX). The homeobox DNA-binding domain is a 60amino-acid motif that binds to DNA via a helix-turn-helix structure, thereby playing 
a role in transcriptional regulation of gene expression. PRRX1b shares most of the sequence with PRRX1a except the OAR domain. ${ }^{4}$

PRRX1 usually acts as a transcription factor or transcriptional coactivator to regulate the expression of target genes. Several DNA sequences that PRRX1 can bind to have been reported. As a transcription factor, PRRX1 can bond to the promoter sequence (5'-AGCCCTTCACTA CTGTTGACTTCTC-3') of PPARG2 to regulate its expression. ${ }^{5}$ Also, PRRX1b can bond to SOX9 promoter in a highly conserved sequence ( $5^{\prime}$-AACAAATTACA-3'). As a transcription co-activator, PRRX1 can bind to PRRX2 in order to regulate MIR875 at the 5'-AATTA-3' region of its promoter. ${ }^{6}$ Also, PRRX1 can interact with serum response factor (SRF) and SRF-Phox1 interacting protein (SPIN) to bond to the promoter of FOS at the $5^{\prime}$ GTCAAT-3'region. ${ }^{7}$

PRRX1 is abnormally expressed in many diseases and is involved in tumor metastasis. Especially, it is closely correlated to the occurrence of epithelial-mesenchymal transition (EMT) and stemness maintenance of tumor cells, consequently affecting the proliferation and metastasis of cancer cells.

\section{PRRXI Promotes EMT of Tumor Cells}

In 2012, Ocana et al first found that overexpression of PRRX1 could promote EMT in cancer cells. ${ }^{8}$ Recent studies have reported that PRRX1 is involved in the metastasis of many cancers, mainly through regulating the occurrence of EMT, a biological process that enables a polarized epithelial cell to acquire a mesenchymal cell phenotype. ${ }^{9}$ During EMT, epithelial cells lose their polarity and intercellular interaction, and gain abilities of migration, invasion, anti-apoptosis, and degradation of extracellular matrix. EMT, which partly related to the activities of matrix metalloproteinase family is regarded as a hallmark of malignant tumors. MMP2 and MMP9 are two important matrix metalloproteinases that correlated with tumor cell tumor aggressiveness and overall survival. Thus, they are commonly used as serum markers of the malignant phenotype. ${ }^{10}$ Another protein family that linked to EMT is the cadherin family, which include E-cadherin, $\mathrm{N}$-cadherin, etc. More and more studies have proved that PRRX1 can regulate cadherin family to directly regulate the occurrence or process of EMT through the Wnt $/ \beta$ - catenin signaling pathway and/or Notch signaling pathway (Figure 1).

\section{PRRXI Promotes EMT Through Wnt/ $\beta$ - Catenin Signaling Pathway}

In breast and gastric cancer, PRRX1 promotes EMT mainly through the $\mathrm{Wnt} / \beta$-catenin signaling pathway. $\mathrm{LV}$ et al found that the repression of PRRX1b inhibited EMT via the $\mathrm{Wnt} / \beta$-catenin signaling pathway and its downstream target gene Cyclin D1 (CCND1). They speculated that PRRX1b induced EMT through the Wnt/ $\beta$-catenin signaling pathway, leading to invasion and metastasis of tumor cells. ${ }^{11}$ Studies have also proved that PRRX1 activated the $W n t / \beta$-catenin signaling pathway and induces the binding of Wnt molecules to transmembrane protein receptors in gastric cancer, thereby inhibiting the destruction of the complex and allowing the accumulation of $\beta$ catenin into the nucleus. In addition, XAV939, an inhibitor of the Wnt/ $\beta$-catenin signaling pathway, has been shown to attenuate EMT caused by over-expression of PRRX1, consequently inhibiting the invasion and migration of gastric cancer cells. $^{12}$

\section{PRRXI Promotes EMT Through Notch Signaling Pathway}

Studies on the invasion ability of malignant glioblastoma cells have also shown an association with PRRX1. Sugiyama et al found that the expression of PRRX1 in glioblastoma cells was positively correlated with the expression of its target gene Hes family BHLH transcription factor 1 (HES1) in the downstream of the Notch signaling pathway, suggesting that PRRX1 may activate the Notch signaling pathway. ${ }^{13}$ Inhibition of the Notch signaling pathway with a $\gamma$-secretase (DAPT) inhibitor can suppress the PRRX1-promoted cell invasion, further proving that PRRX1 promotes the invasion of malignant gliomas by inducing the Notch signaling pathway. However, knockdown of PRRX1 in U87 and U251sp cells had no effects on the Notch signaling pathway, indicating that a certain cellular environment is required for PRRX1-mediated activation of the Notch signaling pathway. Additionally, the Notch signaling pathway is also regulated by other upstream proteins. For instance, the immunoglobulin kappa J region (RBP-J), a main transcriptional mediator of Notch signaling, can lead to activation of Notch signaling pathway. And lack of RBPJ can 


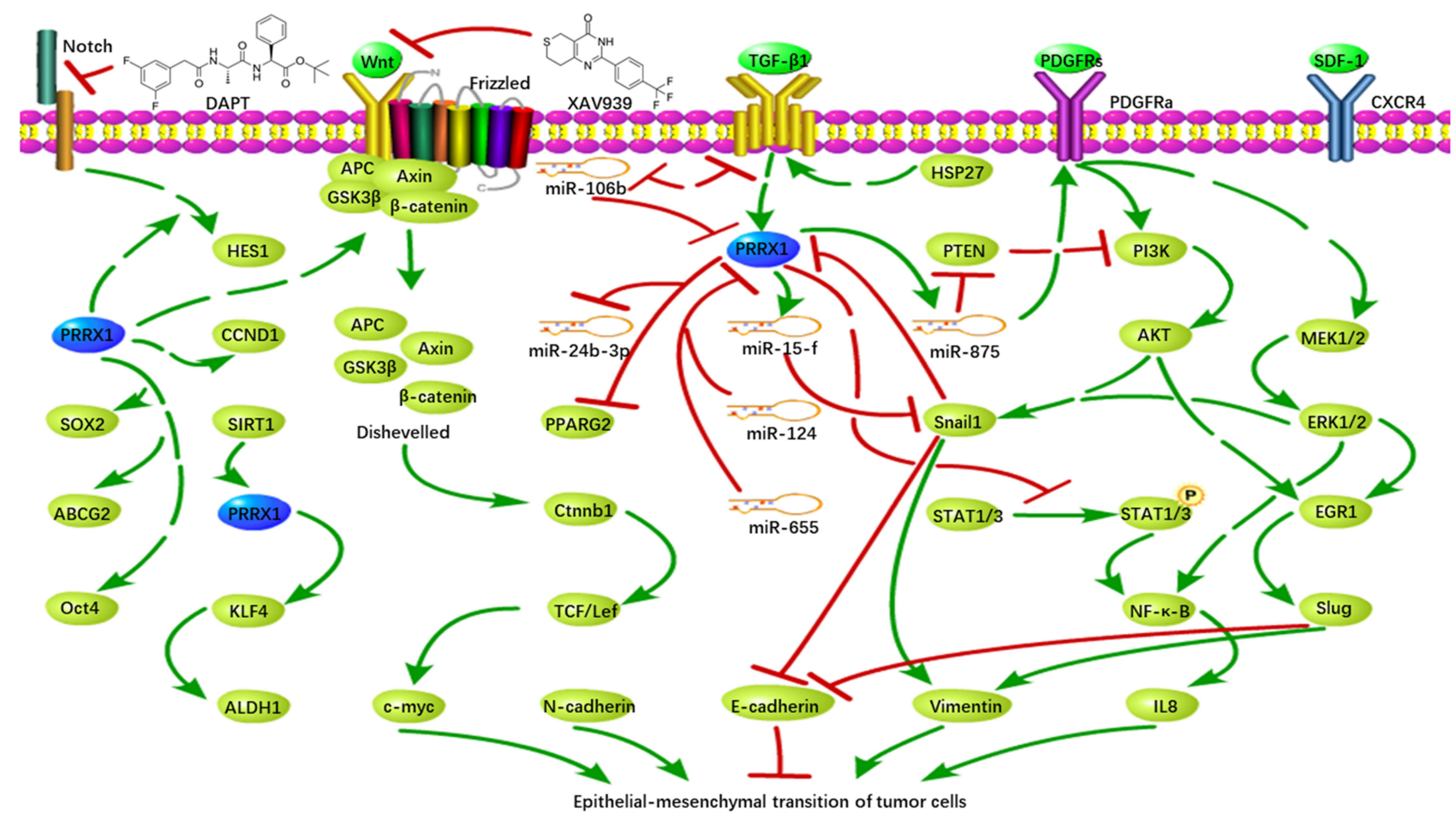

Figure I Regulatory roles of PRRXI in EMT of tumor cells.

suppress the invasion of PRRX1-expressing cells by blocking Notch signaling pathway. ${ }^{13}$

\section{PRRXI Promotes EMT Through Maintaining the Stem of Tumor Cells}

PRRX1 has also been observed to promote EMT through maintaining the stemness of cancer stem cells (CSCs). CSCs are a small group of cells with stem cell characteristics in tumors, which can maintain cancer progression and have the ability to self-renew and regenerate. ${ }^{14}$ Studies have provided evidences to support a direct relationship between EMT and CSC. EMT can confer tumor cells with stem cell-like characteristics, thus increasing the invasion and proliferation of tumor cells. ${ }^{15}$ Moreover, PRRX1 can induce the stemness of colorectal cancer (CRC) cells. ${ }^{16}$ In CRC cells, inhibition of miR-124 upregulates PRRX1, leading to upregulation of stem-related genes ATP binding cassette subfamily G member 2 (ABCG2), SRY-box transcription factor 2 (SOX2), and major octamer-binding protein4 (OCT4). ${ }^{17}$ Other studies have shown that sirtuin 1 (SIRT1) upregulates the cell stemness marker aldehyde dehydrogenase 1 family member A1 (ALDH1) ARX through PRRX1 deacetylation. Elimination of PRRX1 inhibits core cell stem factor Kruppel-like factor 4
(KLF4), and then forms a SIRT1-PRRX1-KLF4-ALDH1 pathway, which enhances the stemness of tumor cells. Meanwhile, the depletion of SIRT1 results in an enhanced epithelial plasticity in basal-like breast cancer cells and induces highly proliferative MET-type ALDH1+ CSCs, as a result promoting metastasis of tumor cells and drug resistance, which may due to the upregulation of ATPbinding cassette subfamily $\mathrm{G}$ member 2 (ABCG2) by PRRX1. ${ }^{18,19}$ Recent studies have demonstrated that leucine-rich repeat-containing $G$ protein-coupled receptor 5 (LGR5)-expressed CD54+ cells have the characteristics of stem cells in gastric cancer cells, and LGR5 is positively correlated with cell stemness and PRRX1 expression. ${ }^{20} \mathrm{Li}$ et al found that the ability of PRRX1 to regulate the selfrenewal of glioma stem cells was regulated by the dopamine D2 (DRD2) receptor, which can stimulate the release of dopamine (DA) to activate the extracellular signalregulated kinase (ERK) and c-Jun NH2-terminal kinase (JNK) pathways. Furthermore, DA promotes mitogenesis, which is closely related to Ras-dependent mitogenactivated protein kinase (MAPK) and JNK pathways, and is associated with tumorigenicity and aggressiveness. ${ }^{21}$ PRRX1 directly activates the DRD2 signaling pathway by binding to the promoter of DRD2 receptor, consequently ensuring the tumorigenicity and invasion of 
glioma stem cells. Knockdown of PRRX1 reduces the expression of DRD2 and its consequent extracellular signal-related kinases ERK and AKT. Thus, DRD2 can regulate the proliferation, differentiation, and metastasis of glioma cells via ERK and PI3K/AKT signaling pathways, providing a theoretical basis for the study of PRRX1 -DRD2-ERK/AKT axis. $^{22}$

\section{The Effects of PRRXI Isoforms on EMT}

Two isoforms, PRRX1a and PRRX1b, have been identified for PRRX1. The difference between PRRX1a and PRRX1b is at their C-terminus. PRRX1a is the canonical PRRX1 transcript that harbors an OAR (otp, aristaless, and rax) domain, which is named after three proteins sharing this $15-$ amino-acid region, while the $\mathrm{C}$ terminal end of PRRX1b does not contain any other known protein domains. ${ }^{4}$ Recent studies have shown that PRRX1a and PRRX1b play different roles in EMT. When the total PRRX1 is silenced, the motility of liver cancer cells is enhanced, leading to invasion and metastasis of cancer cells. ${ }^{23}$ Further investigation demonstrates that PRRX1a stimulates tumor metastasis, differentiation, and mesenchymal-epithelial transformation in the liver. In contrast, PRRX1b promotes the invasion, dedifferentiation, and EMT of cancer cells. Furthermore, both PRRX1a and PRRX1b were associated with the invasion and metastasis of PDC and 4313 pancreatic cancer cells. ${ }^{24} \mathrm{GO}$ analysis demonstrated that PRRX1a markedly promoted tumor cell metastasis, while PRRX1b mainly affected tumor cell invasion. $^{24}$

\section{TGF- $\beta$ Induces EMT Through PRRXI}

Zheng et al found that PRRX1 was the target gene of miR106b. Knockdown of miR-106b in CRC cells increased the expression of PRRX1, which accelerated the process of EMT. $^{25}$ They also found that TGF- $\beta 1$ inhibited the expression of miR-106b, which also had a negative feedback effect on TGF- $\beta 1$. This formed a negative feedback loop that regulated the expression of PRRX1, thereby regulating the occurrence of EMT. ${ }^{25}$ In head and neck squamous cell carcinoma (HNSCC) cells, PRRX1 cooperates with activated TGF- $\beta 1$ to promote the migration and invasion of cancer cells, and subsequently regulates the phenotypic plasticity and dormancy of tumor cells. ${ }^{26}$ In lung cancer, PRRX1a has been found upregulated in lung cancer tissues and correlated with TGF- $\beta$ expression positively. ${ }^{27}$ Also, Chen et al found that PRRX1 knockdown can upregulated paired-like homeodomain 2 (PITX2) in lung cancer tissues. The upregulation of this protein can increase miR- 200a and miR-200b/429, which respectively inhibited CTNNB1 and SLUG, one of the TGF- $\beta$ downstream signals As a result, promoting the effect of E-cadherin. ${ }^{28}$ Hardin et al treated thyroid cancer cells with TGF- $\beta 1$ and found that the expression of PRRX1 was increased, with upregulation of Slug and Snail. Snail can directly bind to the promoter of E-cadherin, thereby inhibiting the expression of E-cadherin, reducing the intercellular interaction, and eventually leading to the occurrence of EMT. ${ }^{29}$ Overexpression of small heat shock protein (HSP27) in salivary adenoid cystic carcinoma cells promotes EMT process through the TGF- $\beta 1$ signaling pathway, accompanied by upregulation of Snail1 and PRRX1. Studies have shown that HSP27 inhibits E-cadherin e-pression by activating Snaill and PRRX1. At the same time, overexpression of Snail1 and PRRX1 increased migratory capacity and invasiveness of cancer cells. ${ }^{30}$ Fazilaty et al further revealed the interaction between Snaill and PRRX1, which showed that Snaill acted as a repressor to inhibit the transcription of PRRX1. However, PRRX1 promoted its own transcription and induced miR-15f to indirectly inhibit the expression of Snail1. Although both of them were activated by TGF- $\beta 1$, the promoter of Snaill was more sensitive to the signal and therefore was expressed preferentially, thereby inhibiting PRRX1 expression and subsequent epithelial cells interactions with surrounding cells. $^{31}$

\section{miRNA Regulates EMT Through PRRXI}

Zhang et al found that PRRX1 was a direct target gene of miR-124, ${ }^{17}$ which was also confirmed by Lin et al. ${ }^{32}$ Overexpression of miR-124 reduced the expression of PRRX1, consequently increased the sensitivity of CRC cells to radiation. In triple-negative breast cancer (TNBC), miR-655 suppresses EMT through direct inhibition of PRRX1 expression. ${ }^{33}$ In addition, the expression of PRRX1 in HNSCC cells was significantly negatively correlated with miR-642b-3p, and its expression was downregulated by overexpression of PRRX1. Reinforced expression of miR$642 \mathrm{~b}-3 \mathrm{p}$ reversed the PRRX1-promoted EMT and proliferation of cancer cells. ${ }^{26}$ Funada et al found that PRRX1 could enhance the expression of miR-875 by binding to its promoter region. MiR-875, which is proved as an EMT inducer, can promote cell migration through the PDGF signaling pathway. In melanoma progression, PRRX1 was found upregulated and negatively correlated with miR-485-5p. ${ }^{6} \mathrm{Wu}$ et al found that miR-485-5p can target PRRX1 to silence its expression. As a result, inhibiting the progress of EMT. ${ }^{34}$ 


\section{The Functional Mechanisms of PRRXI in Tumor}

Studies have demonstrated that PRRX1 plays a role as a transcription factor or transcriptional co-activator during tumorigenesis (Figure 2)

\section{PRRXI Functions as a Transcription Factor}

As a transcription factor, PRRX1 binds to the promoter of the target genes to stimulate or inhibit their transcription, which consequently promotes the proliferation and/or invasion of tumor cells. Some EMT-related genes have been reported to be regulated by PRRX1. For instance, PRRX1 directly binds to the promoter of PPARG2 and inhibits its expression, which promotes the progression of EMT. ${ }^{5}$ Meanwhile, PRRX1b, an alternative splicing isoform of PRRX1, is able to bind to the promoter of HGF to stimulate the HGF/MET signaling pathway, which is an important pathway to stimulate EMT and tumor growth. ${ }^{24}$ PRRX1 also promotes the proliferation of tumor cells by acting as a transcription factor of relevant genes. Reichert et al revealed that PRRX1b bonded to SOX9 promoter at the region between -1950 and -1830 nucleotides (nts), which promoted the expression of SOX9 and led to cell migration. They also found that a highly conserved PRRX1 DNA- binding sequence in this region was able to bind to PRRX1b. ${ }^{4}$ It has also been reported that PRRX1 can bind to the promoter region of dopamine receptor D2 (DRD2) gene and transactivate the expression of DRD2 in gastrointestinal cancer cells, resulting in the proliferation of tumor cells. Specifically, Li et al demonstrated that PRRX1 binds to the upstream of the DRD2 gene ( -281 to $-173 \mathrm{nt})$ using luciferase reporter assay and ChIP-qPCR. ${ }^{22}$

In addition, PRRX1 contributes to the development of tumor by binding to the promoter of miRNA gene or miRNA precursor and consequently regulating miRNA expression. For instance, PRRX1 can bind to the promoter region of MIR875 at -500 to +100 nts, especially the sequence of "TAATTA" at about -192 nts, which stimulates the maturation of miR-875-5p and promotes the migration of tumor cells. ${ }^{6}$ Interestingly, Jiang et al found that PRRX1 bonded to the hypoxia response element regions of pre-miR-642b to inhibit the maturation of miR-642b-3p, which inhibited the EMT of tumor cells. ${ }^{26}$

\section{PRRXI Functions as a Transcription Co-Activator}

Studies also provide evidences to support the transcription co-activator roles of PRRX1 by binding to transcription factors or other proteins. For instance, PRRX1 binds to the

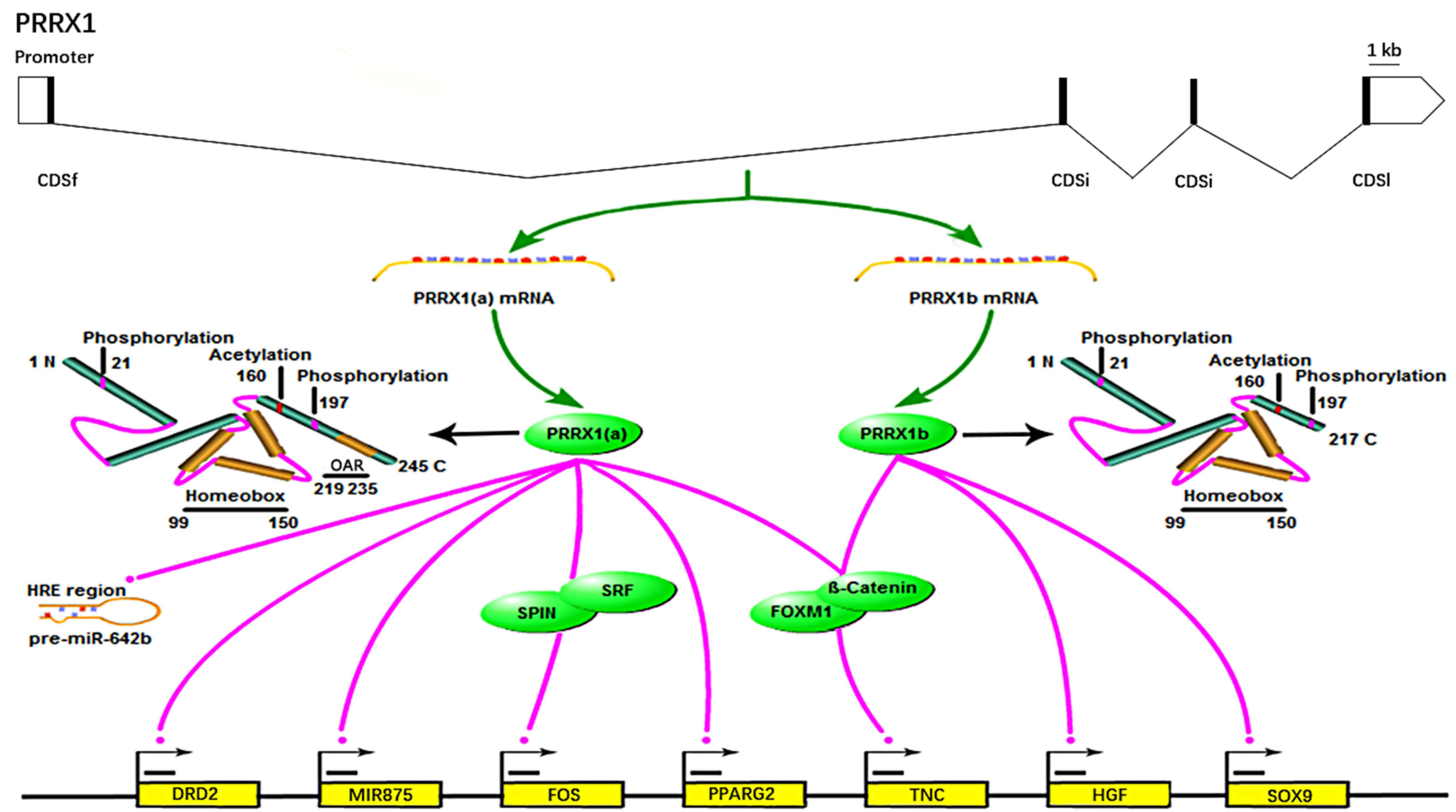

Figure 2 Transcriptional roles of PRRXI in tumorigenesis. 
Forkhead domain of Forkhead Box M1 (FOXM1) to increase the stability and transcriptional activity of FOXM1, leading to the upregulation of target genes. ${ }^{35}$ Grueneberg et al reported that PRRX1 interacted with SRF to enhance serum responsive a result, promoting tumor progression. ${ }^{7}$

\section{Summary}

PRRX1 is an important member of the paired family of homeobox proteins localized to the nucleus of the cell. It is a transcription activator involved in tumor metastasis. ${ }^{8}$ In particular, it plays a significant role in the occurrence of EMT and the maintenance of tumor cell stemness, and affects the proliferation and metastasis of tumor cells. The mechanisms of PRRX1 promoting EMT are different in different tissues and types of cancer. PRRX1 promotes EMT through Wnt/ $\beta$-catenin signaling pathway, Notch signaling pathway, and maintenance of tumor stem cells. In addition, there are close relationships between PRRX1, TGF- $\beta$, mi-RNA and EMT. Feldmann et al found that PRRX1 in cancer-associated fibroblasts (CAFs) promoted EMT and chemotherapeutic resistance in tumor cells through paracrine hepatocyte growth factor (HGF) signaling. ${ }^{36}$ However, PRRX1 has also been found to inhibit the occurrence of EMT of cancer cells. For instance, PRRX1 knockdown induces the occurrence of EMT with decreased cadherin 1 (CDH1) and increased cadherin 2 (CDH2) and Vimentin (VIM), resulting in invasion and metastasis of lung cancer cells. $^{37}$ Moreover, inhibition of PRRX1 also enhanced the anti-apoptotic and resistant abilities of A549 cells to cisplatin. $^{38}$ In liver cancer, SDF-1 can induce EMT and cell stemness. PRRX1 is negatively regulated by SDF-1. While inhibition of PRRX1 stimulates the receptor of Stromal Cell Derived Factor-1 (SDF-1), C-X-C motif chemokine receptor 4 (CXCR4), thereby enhancing the regulatory effects of SDF-1 on cells. At the same time, inhibition of PRRX1 can phosphorylate signal transducer andactivator of transcription 3 (STAT3) and strengthen the invasion ability of liver cancer cells. ${ }^{39}$ Since PRRX1 has a strong relationship with different kinds of tumor, further study on the mechanism of PRRX1 will provide a theoretical basis to elucidate the formation and development of tumor, and provide a new target for tumor treatment. Also, there are several microRNAs that regulate PRRX1 in different tumor tissues, micronucleic acid drugs targeting PRRX1 may be a new kind of cure for cancer.

\section{Disclosure}

The authors report no conflicts of interest in this work.

\section{References}

1. Graba Y, Aragnol D, Pradel J. Drosophila Hox complex downstream targets and the function of homeotic genes. BioEssays. 1997;19:379-388. doi:10.1002/bies.950190505

2. Garcia-Bellido A. Genetic control of wing disc development in Drosophila. Ciba Foundation symposium; 1975:161-182.

3. Dettman RW, Steinhorn RH. Connecting the cells - vascular differentiation via homeobox genes and extracellular matrix in the distal lung. CircRes. 2004;94:1406-1407.

4. Reichert M, Takano S, von Burstin J, et al. The Prrx1 homeodomain transcription factor plays a central role in pancreatic regeneration and carcinogenesis. Genes Dev. 2013;27:288-300. doi:10.1101/gad.20 4453.112

5. Jiang YP, Tang YL, Wang SS, et al. PRRX1-induced epithelial-tomesenchymal transition in salivary adenoid cystic carcinoma activates the metabolic reprogramming of free fatty acids to promote invasion and metastasis. Cell Prolif. 2020;53:e12705. doi:10.1111/cpr.12705

6. Funada K, Yoshizaki K, Kanako M, et al. microRNA-875-5p plays critical role for mesenchymal condensation in epithelial-mes enchymal interaction during tooth development. Sci Rep. 2020;10:4918. doi:10.1038/s41598-020-61693-w

7. Grueneberg DA, Henry RW, Brauer A, et al. A multifunctional DNA-binding protein that promotes the formation of serum response factor/homeodomain complexes: identity to TFII-I. Genes Dev. 1997;11:2482-2493. doi:10.1101/gad.11.19.2482

8. Ocaña $\mathrm{OH}$, Córcoles R, Fabra A, et al. Metastatic colonization requires the repression of the epithelial-mesenchymal transition inducer Prrx1. Cancer Cell. 2012;22(6):709-724. PMID: 23201163. doi:10.1016/j.ccr.2012.10.012

9. Kalluri R, Weinberg RA. The basics of epithelial-mesenchymal transition. J Clin Invest. 2009;119:1420-1428. doi:10.1172/JCI39104

10. Di Carlo A, Mariano A, Terracciano D, et al. Matrix metalloproteinase-2 and -9 in the urine of prostate cancer patients. Oncol Rep. 2010;24:3-8. doi:10.3892/or_00000821

11. Lv ZD, Yang ZC, Liu XP, et al. Silencing of Prrx $1 b$ suppresses cellular proliferation, migration, invasion and epithelial-mesenchymal transition in triple-negative breast cancer. J Cell Mol Med. 2016;20:1640-1650. doi: $10.1111 / \mathrm{jcmm} .12856$

12. Guo JB, Fu ZX, Wei JL, Lu WD, Feng JH, Zhang SR. PRRX1 promotes epithelial-mesenchymal transition through the Wnt/beta-catenin pathway in gastric cancer. Med Oncol. 2015;32:12. doi:10.1007/ s12032-014-0393-x

13. Sugiyama M, Hasegawa H, Ito S, et al. Paired related homeobox 1 is associated with the invasive properties of glioblastoma cells. Oncol Rep. 2015;33:1123-1130. doi:10.3892/or.2014.3681

14. Aponte PM, Caicedo A. Stemness in cancer: stem cells, cancer stem cells, and their microenvironment. Stem Cells Int. 2017;5619472:2017.

15. Mani SA, Guo W, Liao MJ, et al. The epithelial-mesenchymal transition generates cells with properties of stem cells. Cell. 2008;133:704-715. doi:10.1016/j.cell.2008.03.027

16. Takahashi Y, Sawada G, Kurashige J, et al. Paired related homoeobox 1, a new EMT inducer, is involved in metastasis and poor prognosis in colorectal cancer. Br J Cancer. 2013;109:307-311. doi:10.1038/ bjc.2013.339

17. Zhang Y, Zheng L, Huang J, et al. MiR-124 radiosensitizes human colorectal cancer cells by targeting PRRX1. PLoS One. 2014;9: e93917. doi:10.1371/journal.pone.0093917

18. Němcová-Fürstová $\mathrm{V}$, Kopperová $\mathrm{D}$, Balušíková $\mathrm{K}$, et al. Characterization of acquired paclitaxel resistance of breast cancer cells and involvement of $\mathrm{ABC}$ transporters. Toxicol Appl Pharmacol. 2016;310:215-228. doi:10.1016/j.taap.2016.09.020 
19. Shi L, Tang XL, Qian MX, et al. A SIRT1-centered circuitry regulates breast cancer stemness and metastasis. Oncogene. 2018;37:6299-6315. doi:10.1038/s41388-018-0370-5

20. Wang B, Chen QT, Cao Y, et al. LGR5 is a gastric cancer stem cell marker associated with stemness and the EMT signature genes NANOG, NANOGP8, PRRX1, TWIST1, and BMI1. PLoS One. 2016;11:21. doi:10.1371/journal.pone.0168904

21. Luo Y, Kokkonen GC, Wang X, Neve KA, Roth GS. D2 dopamine receptors stimulate mitogenesis through pertussis toxin-sensitive $\mathrm{G}$ proteins and Ras-involved ERK and $\mathrm{SAP} / \mathrm{JNK}$ pathways in rat C6-D2L glioma cells. J Neurochem. 1998;71:980-990. doi:10.1046/ j.1471-4159.1998.71030980.x

22. Li Y, Wang W, Wang FY, et al. Paired related homeobox 1 transactivates dopamine D2 receptor to maintain propagation and tumorigenicity of glioma-initiating cells. J Mol Cell Biol. 2017;9:302-314. doi: $10.1093 / \mathrm{jmcb} / \mathrm{mjx} 017$

23. Fan M, Shen J, Liu H, et al. Downregulation of PRRX1 via the p53-dependent signaling pathway predicts poor prognosis in hepatocellular carcinoma. Oncol Rep. 2017;38:1083-1090. doi:10.3892/ or.2017.5785

24. Takano S, Reichert M, Bakir B, et al. Prrx1 isoform switching regulates pancreatic cancer invasion and metastatic colonization. Genes Dev. 2016;30:233-247. doi:10.1101/gad.263327.115

25. Zheng L, Zhang YQ, Lin SM, et al. Down-regualtion of miR-106b induces epithelial-mesenchymal transition but suppresses metastatic colonization by targeting Prrx1 in colorectal cancer. Int J Clin Exp Pathol. 2015;8:10534-U14336.

26. Jiang J, Zheng M, Zhang M, et al. PRRX1 regulates cellular phenotype plasticity and dormancy of head and neck squamous cell carcinoma through miR-642b-3p. Neoplasia (New York, NY). 2019; 21:216-229. doi:10.1016/j.neo.2018.12.001

27. Sun L, Zhang X, Liu X, et al. PRRX1 isoform PRRX1A regulates the stemness phenotype and epithelial-mesenchymal transition (EMT) of cancer stem-like cells (CSCs) derived from non-small cell lung cancer (NSCLC). Transl Lung Cancer Res. 2020;9(3):731-744. doi:10.21037/tlcr-20-633

28. Chen W, Wu J, Shi W, et al. PRRX1 deficiency induces mesenchymal-epithelial transition through PITX2/miR-200-dependent SLUG/CTNNB1 regulation in hepatocellular carcinoma. Cancer Sci. 2021;112(6):2158-2172. doi:10.1111/cas.14853
29. Hardin H, Guo ZY, Shan WH, et al. The roles of the epithelial-mesenchymal transition marker PRRX1 and miR-146b-5p in papillary thyroid carcinoma progression. Am J Pathol. 2014;184:2342-2354. doi:10.1016/j.ajpath.2014.04.011

30. Chen W, Ren XH, Wu JS, et al. HSP27 associates with epithelial-mesenchymal transition, stemness and radioresistance of salivary adenoid cystic carcinoma. J Cell Mol Med. 2018;22: 2283-2298. doi:10.1111/jcmm.13510

31. Fazilaty H, Rago L, Kass Youssef K, et al. A gene regulatory network to control EMT programs in development and disease. Nat Commun. 2019;10:5115. doi:10.1038/s41467-019-13091-8

32. Lin SM, Xia Q, Zhang YQ, et al. [miR-124 regulates radiosensitivity of colorectal cancer cells by targeting PRRX1]. Nan Fang Yi Ke Da Xие Xие Bao. 2016;36:1110-1116. (Chinese).

33. Lv ZD, Kong B, Liu XP, et al. miR-655 suppresses epithelial-tomesenchymal transition by targeting Prrx 1 in triple-negative breast cancer. J Cell Mol Med. 2016;20:864-873. doi:10.1111/jcmm.12770

34. Wu X, Bao H. Tumor suppressive microRNA-485-5p targets PRRX1 in human skin melanoma cells, regulating epithelial-mesenchymal transition and apoptosis. Cell Biol Int. 2021;45(7):1404-1414. doi:10.1002/cbin. 11575

35. Marchand B, Pitarresi JR, Reichert M, Suzuki K, Laczkó D, Rustgi AK. PRRX1 isoforms cooperate with FOXM1 to regulate the DNA damage response in pancreatic cancer cells. Oncogene. 2019;38:4325-4339. doi:10.1038/s41388-019-0725-6

36. Feldmann K, Maurer C, Peschke K, et al. Mesenchymal plasticity regulated by Prrx1 drives aggressive pancreatic cancer biology. Gastroenterology. 2021;160(1):346-361.e24. PMID: 33007300. doi:10.1053/j.gas-tro.2020.09.010

37. Zhu HB, Sun GY. Loss of PRRX1 induces epithelial-mesenchymal transition and cancer stem cell-like properties in A549 cells. Am J Transl Res. 2017;9:1641-1650.

38. Zhu HB, Sun GY, Dong JH, Fei LM. The role of PRRX1 in the apoptosis of A549 cells induced by cisplatin. Am J Transl Res. 2017;9:396-402.

39. Tang Y, Lu Y, Chen Y, et al. Pre-metastatic niche triggers SDF-1/ CXCR4 axis and promotes organ colonisation by hepatocellular circulating tumour cells via downregulation of Prrx1. J Exp Clin Cancer Res. 2019;38:473. doi:10.1186/s13046-019-1475-6
OncoTargets and Therapy

\section{Publish your work in this journal}

OncoTargets and Therapy is an international, peer-reviewed, open access journal focusing on the pathological basis of all cancers, potential targets for therapy and treatment protocols employed to improve the management of cancer patients. The journal also focuses on the impact of management programs and new therapeutic

Submit your manuscript here: https://www.dovepress.com/oncotargets-and-therapy-journa agents and protocols on patient perspectives such as quality of life, adherence and satisfaction. The manuscript management system is completely online and includes a very quick and fair peer-review system, which is all easy to use. Visit http://www.dovepress.com/ testimonials.php to read real quotes from published authors. 